\title{
Molecular diagnostic experience of whole-exome sequencing in adult patients
}

\author{
Jennifer E. Posey, MD, PhD'1, Jill A. Rosenfeld, MS1', Regis A. James, BS², Matthew Bainbridge, PhD ${ }^{1,3}$, \\ Zhiyv Niu, PhD ${ }^{1,4}$, Xia Wang, PhD1 ${ }^{1}$, Shweta Dhar, MD ${ }^{1,5}$, Wojciech Wiszniewski, MD, PhD1, \\ Zeynep H.C. Akdemir, PhD1, Tomasz Gambin, PhD1, Fan Xia, PhD ${ }^{1,4}$, Richard E. Person, PhD ${ }^{1,4}$, \\ Magdalena Walkiewicz, PhD ${ }^{1,4}$, Chad A. Shaw, PhD'1, V. Reid Sutton, MD ${ }^{1}$, Arthur L. Beaudet, MD ${ }^{1}$, \\ Donna Muzny, MS ${ }^{1,3}$, Christine M. Eng, MD ${ }^{1,4}$, Yaping Yang, PhD ${ }^{1,4}$, Richard A. Gibbs, PhD ${ }^{1,3,4}$, \\ James R. Lupski, MD, PhD ${ }^{1,3,6,7}$, Eric Boerwinkle, PhD ${ }^{3,8}$ and Sharon E. Plon, MD, PhD $1,3,6,7,9$
}

Purpose: Whole-exome sequencing (WES) is increasingly used as a diagnostic tool in medicine, but prior reports focus on predominantly pediatric cohorts with neurologic or developmental disorders. We describe the diagnostic yield and characteristics of WES in adults.

Methods: We performed a retrospective analysis of consecutive WES reports for adults from a diagnostic laboratory. Phenotype composition was determined using Human Phenotype Ontology terms.

Results: Molecular diagnoses were reported for $17.5 \%$ (85/486) of adults, which is lower than that for a primarily pediatric population $(25.2 \% ; P=0.0003)$; the diagnostic rate was higher $(23.9 \%)$ for those $18-30$ years of age compared to patients older than 30 years $(10.4 \%$; $P=0.0001)$. Dual Mendelian diagnoses contributed to $7 \%$ of diagnoses, revealing blended phenotypes. Diagnoses were more frequent

\section{INTRODUCTION}

Since its earliest described use 7 years ago, sequencing and analysis of individual genomes have become powerful tools for studying human genomic variation ${ }^{1-4}$ and identifying the cause of disease traits such as clinical neuropathy and inherited forms of hypertension. ${ }^{5,6}$ Genomic studies have demonstrated both the clinical and research utility of whole-exome sequencing (WES) for detecting known and novel mutations in disease-causing genes across a variety of inheritance patterns. ${ }^{7-9}$ Clinically, genome-scale sequencing often follows exhaustive clinical evaluations unable to conclude an etiologic diagnosis. Genomic studies have proven especially useful for conditions with locus heterogeneity (long molecular differentials) or unexpected phenotypic variation. ${ }^{10}$ WES also allows for identification of pathologic variants in newly identified disease genes and future re-analysis of existing genomic data. ${ }^{11-13}$ among individuals with abnormalities of the nervous system, skeletal system, head/neck, and growth. Diagnostic rate was independent of family history information, and de novo mutations contributed to $61.4 \%$ of autosomal dominant diagnoses.

Conclusion: Early WES experience in adults demonstrates molecular diagnoses in a substantial proportion of patients, informing clinical management, recurrence risk, and recommendations for relatives. A positive family history was not predictive, consistent with molecular diagnoses often revealed by de novo events, informing the Mendelian basis of genetic disease in adults.

Genet Med advance online publication 3 December 2015

Key Words: adult patients; whole-exome sequencing

The majority of diagnostic WES referrals are pediatric, with adult probands constituting just $16.2,{ }^{14} 36.1,{ }^{15}$ and $12.2 \%{ }^{11}$ of reported clinical cohorts. Several case reports and small studies have described the clinical utility of WES in adults, particularly in cases where an atypical phenotype or polygenic burden may confound a primary diagnosis, ${ }^{16-20}$ but no large-scale analyses of the use of WES by physicians caring for adult patients have been reported. To further explore the nature of genetic disease in adults, we describe indications for testing and diagnostic yield of clinical WES in adult patients referred by their physician to a single academic diagnostic laboratory.

\section{MATERIALS AND METHODS}

\section{Patient population}

We performed a retrospective review of diagnostic WES in the Whole Genome Laboratory at Baylor College of Medicine for a

The first two authors contributed equally to this work.

${ }^{1}$ Department of Molecular and Human Genetics, Baylor College of Medicine, Houston, Texas, USA; ${ }^{2}$ Program in Structural and Computational Biology and Molecular Biophysics, Baylor College of Medicine, Houston, Texas, USA; ${ }^{3}$ Human Genome Sequencing Center, Baylor College of Medicine, Houston, Texas, USA; ${ }^{4}$ Baylor Miraca Genetics Laboratories, Baylor College of Medicine, Houston, Texas, USA; ${ }^{5}$ Department of Medicine, Baylor College of Medicine, Houston, Texas, USA; ${ }^{6}$ Department of Pediatrics, Texas Children's Hospital, Houston, Texas, USA; ${ }^{7}$ Department of Pediatrics, Baylor College of Medicine, Houston, Texas, USA; ${ }^{8}$ Human Genetics Center, University of Texas Health Science Center, Houston, Texas, USA; ${ }^{9}$ Texas Children’s Cancer Center, Texas Children’s Hospital, Houston, Texas, USA. Correspondence: Sharon E. Plon (splon@bcm.edu) 
3-year period (October 2011-November 2014), including tests from a large number of United States and international medical institutions. Cancer exomes, offered under a separate test code, were not included in this analysis. In all cases, WES was performed on the proband sample only (referred to here as proband-WES); although parental WES was not performed, available parental samples were obtained for variant confirmation by Sanger analysis. Of 4,476 individuals with diagnostic proband-WES, 505 were 18 years or older at the time of referral. After excluding healthy adults and affected related individuals, 486 adults met criteria for study inclusion. The institutional review board at Baylor College of Medicine approved de-identified reporting of demographic and molecular data from this laboratory.

\section{WES and variant analysis}

Library construction, exome capture using VCRome version 2.1 (Roche NimbleGen, Madison WI) ${ }^{21}$ HiSeq next-generation sequencing, and data processing were performed as previously described. ${ }^{12}$ The diagnostic WES evaluation included a coding single-nucleotide polymorphism array performed for quality control and mitochondrial genome sequencing after PCR amplification. The diagnostic reports described here were finalized prior to the laboratory's implementation of an algorithm for copy-number variant identification using data from exome sequencing. Thus, the diagnostic rate reported here does not include additional diagnoses that might result from identification of exonic deletions or duplications using exome data. A molecular diagnosis required pathogenic or likely pathogenic variants in Mendelian disease genes consistent with the observed phenotype and expected inheritance. ${ }^{11,12}$ Variant classification was performed as previously described and consistent with guidelines set forth by the American College of Medical Genetics and Genomics (ACMG). ${ }^{22}$ Variants described in Supplementary Table S1 online have been submitted to ClinVar (accession numbers SCV000245445 through SCV000245563).

\section{Data analyses}

Basic demographic information, ordering physician specialty, diagnostic indication, and family history were obtained from submitted clinical information. Primary and secondary findings were based on the clinical reports and addenda issued as of 1 July 2015. Patients who declined to receive reports of medically actionable secondary findings were excluded from secondary finding analyses.

Based on available clinical information, Human Phenotype Ontology (HPO) terms ${ }^{23}$ were designated for each subject, which allowed assignment of each case to one or more HPO phenotypic abnormality classes. Diagnostic rates and relative frequency of each phenotype class were determined computationally by analysis of HPO terms.

\section{Statistical analyses}

$P$ values for male:female ratios were determined using a twotailed binomial test. Testing was also performed for association between phenotypic class and diagnostic rate. Because individual subjects can have multiple phenotypes, and because some phenotypic groups are sparsely represented, we adopted a permutation testing approach to determine the null distribution of test statistics and corresponding $P$ values. We performed 10,000 Monte Carlo draws for which the overall diagnostic rate (defined as the number of diagnosed and undiagnosed cases) was held constant and subjects were randomly permuted with respect to diagnostic status; the ensemble of phenotypes for each individual case was retained. Analyses comprised the omnibus test considering all 22 phenotype classes at once against solved/unsolved status and for each individual class when assessed against the collapse of all other classes $(2 \times 2$ table). A chi-square test statistic was determined for each twoway table, and $P$ values were determined by counting the number of realizations in which the value was equal or greater than the corresponding value for the study data and dividing by the number of permutations performed.

\section{Demographics}

\section{RESULTS}

Adults, defined as individuals 18 years of age and older, comprised $11.3 \%(505 / 4,476)$ of all diagnostic WES cases in our clinical laboratory between October 2011 and November 2014. After eliminating related individuals or those referred without a clinical indication, 486 were included in the present analysis; 272 of these individuals were included in our previously published reports. ${ }^{11,12}$ WES for adults was ordered by 229 independent physicians; the majority of cases were sent by geneticists

Table 1 Specialties of physicians referring adult patients for whole-exome sequencing

\begin{tabular}{|lcc|} 
Specialty & $\begin{array}{c}\text { Number of } \\
\text { referrals }\end{array}$ & $\begin{array}{c}\text { Percentage } \\
\text { of referrals }\end{array}$ \\
\hline Genetics & 309 & 61.2 \\
\hline Neurology & 111 & 22.0 \\
\hline Neurogenetics & 31 & 6.1 \\
\hline Endocrinology & 12 & 2.4 \\
\hline Rheumatology & 7 & 1.4 \\
\hline Primary care & 6 & 1.1 \\
\hline Hematology & 4 & $<1$ \\
\hline Gastroenterology & 4 & $<1$ \\
\hline Cardiology & 3 & $<1$ \\
\hline Obstetrics/gynecology & 3 & $<1$ \\
\hline Oncology & 3 & $<1$ \\
\hline Ophthalmology & 2 & $<1$ \\
\hline Immunology & 2 & $<1$ \\
\hline Toxicology & 1 & $<1$ \\
\hline Urgent care & 1 & $<1$ \\
\hline Urology & 1 & $<1$ \\
\hline Unknown & 5 & $<1$ \\
\hline Total & 505 & \\
\hline
\end{tabular}



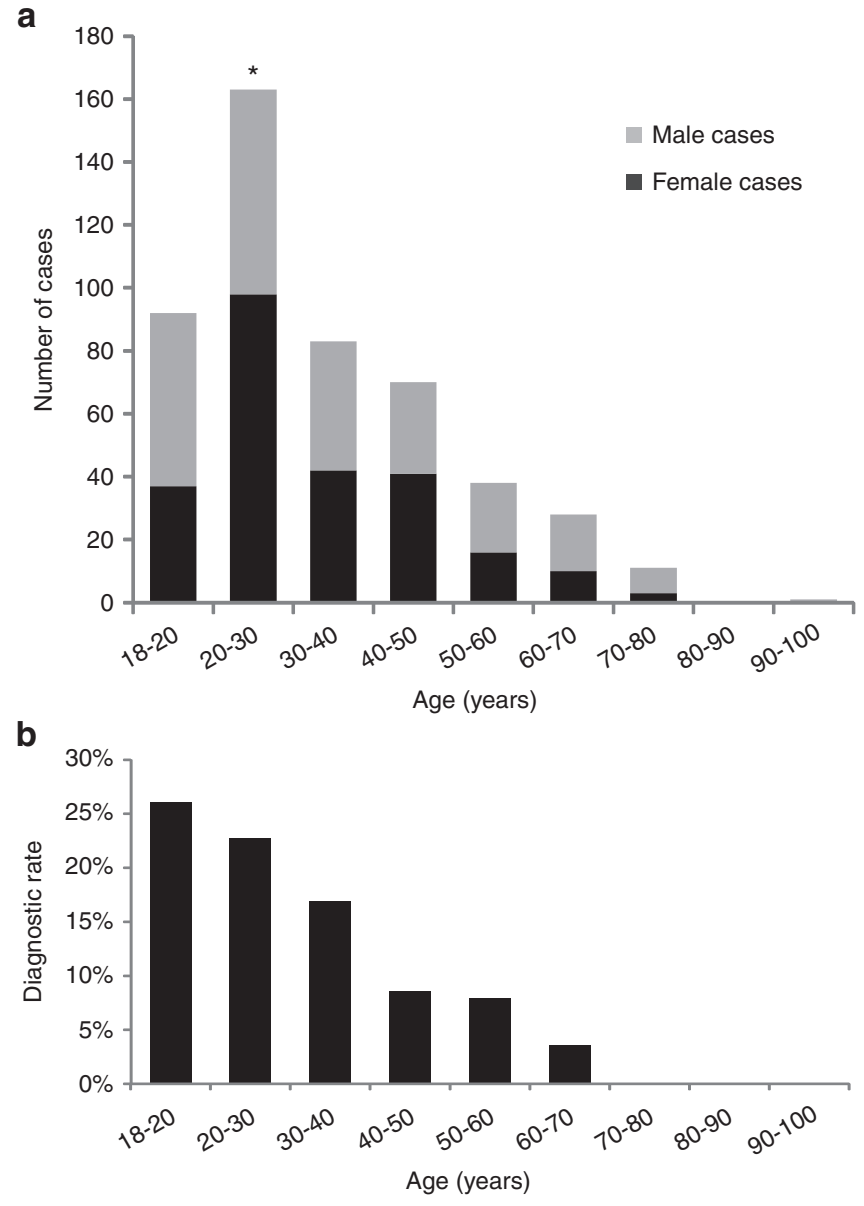

Figure 1 Age and sex of individuals undergoing whole-exome sequencing. (a) Total number of female (black) and male (light gray) cases by age group. ${ }^{*} P<0.05$ for significance of differences between male and female proportions within each age group. (b) Molecular diagnostic rate as percentage of total cases in each age group.

(61.2\%), followed by neurologists $(22.0 \%)$ and those with both neurology and genetics training (6.1\%; Table 1$)$.

Most adult patients were $18-30$ years of age (52.5\%, 255/486), and only $2.5 \%(12 / 486)$ were older than 70 years of age (Figure 1a). Males $(n=239)$ and females $(n=247)$ were equally represented overall $(P=0.75$, binomial test $)$ and within all age ranges, except the predominantly female 20 - to 30 -year-old group $(P=0.01$, binomial test; Figure 1a). Ethnic or racial background was provided by the referring physician in 414 cases, of which mixed European Caucasian descent was indicated for $71.7 \%$, African American was indicated for 3.6\%, Hispanic was indicated for $12.6 \%$ of individuals, and mixed ethnic descent was indicated for $6.0 \%$. Parental consanguinity was reported in 22 (4.5\%) probands. Although WES was not performed on parental samples for the reported cases, both parental samples were available for Sanger analysis of selected variants in $52 \%$ of cases.

\section{Molecular diagnoses}

A molecular (genetic) diagnosis was reported in $85 / 486$ (17.5\%) adults (Supplementary Table S1 online), including six individuals who received two molecular diagnoses each (approximately $7 \%$ of all molecularly diagnosed cases). The molecular diagnostic rate in adults is significantly lower than that observed in a primarily pediatric population $(25.2 \%$, twotailed $P=0.0003$, Fisher exact test). ${ }^{11}$ When stratified by age group, diagnostic rates were higher $(23.9 \%, 61 / 255)$ in cases between 18 and 30 years of age, but declined to $10.4 \%$ (24/231, two-tailed $P=0.0001$, Fisher exact test) in cases older than 30 years of age (Figure 1b). The diagnostic rate was similar when WES was ordered by geneticists $(56 / 296,18.9 \%$; $95 \%$ confidence interval [CI]: 14.87-23.77\%) or neurologists and neurogeneticists (26/139, 18.7\%; 95\% CI: 12.22-25.18\%). All other specialties combined had a low diagnostic rate but represent a small number of cases (3/51, 5.9\%; 95\% CI: 0-12.37\%).

Although a majority of the molecular diagnoses were in nuclear genes, mitochondrial genome sequencing included in the WES test yielded three diagnoses (one individual with two overlapping large mitochondrial deletions and one missense mutation each in MT-ATP6 and MT-ND6; Supplementary Table S1 online). Single-nucleotide polymorphism array data identified one $1.55-\mathrm{Mb}$ pathologic deletion copy-number variant on Xq23 including PLS3 in a male (case 70), one 4.5-Mb deletion on 15q11.2-q13 providing a diagnosis of Angelman syndrome (case 68 ), and one 1.38 -Mb deletion on $17 \mathrm{q} 12$ including $H N F 1 B$ (case 33). The remaining diagnoses were based on a total of 111 distinct variants reported from WES data, of which 60 were novel at the time of reporting (Supplementary Table S1 online). Single-nucleotide variants (SNVs) comprised $75.7 \%(84 / 111)$ of the variants, similar to $76.7 \%$ (543/708, two-tailed $P=0.81$, Fisher exact test) in a primarily pediatric series, ${ }^{11}$ resulting in 57 missense, 16 nonsense, 10 splice site, and 1 initiation codon mutation. Short insertion or deletion variants (indels) comprised $23.4 \%$ (26/111) of diagnostic variants compared to $22.2 \%$ (157/708, two-tailed $P=0.81$, Fisher exact test) in a primarily pediatric series. ${ }^{11}$ Of note, the diversity of underlying Mendelian disorders is demonstrated by only four genes (DYRK1A, FLG, BRAF, and NSD1) with variants reported in two or more unrelated individuals accounting for a total of nine (10.6\%) molecularly diagnosed cases.

The reported mode of inheritance of molecular diagnoses was most frequently autosomal dominant $(48.4 \%)$, followed by autosomal recessive $(40.7 \%)$, X-linked (7.7\%), and mitochondrial (3.3\%; Table 2$)$. This is similar to a primarily pediatric population, ${ }^{11}$ where $53.1 \%(280 / 527$, two-tailed $P=0.43$, Fisher exact test) of diagnoses were autosomal dominant, $34.3 \%$ $(181 / 527, P=0.28)$ were autosomal recessive, $12.3 \%(65 / 527$, $P=0.29)$ were $\mathrm{X}$-linked, and $0.2 \%$ were mitochondrial inheritance. A majority of both autosomal dominant and recessive syndromes included neurologic, muscular/musculoskeletal, or multiple affected organ systems features (Supplementary Table S1 online). Considering all dominant molecular diagnoses, $75.0 \%$ (33/44) were de novo events and/or novel (not previously described) variants in disease genes.

The 37 autosomal recessive molecular diagnoses included only 11 homozygous disease alleles (29.7\%, 11/37; Table 2$)$. 
Table 2 Modes of inheritance observed across 91 molecular diagnoses in 85 cases

\begin{tabular}{lcc} 
Mode of inheritance & $\begin{array}{c}\text { Number of } \\
\text { diagnoses }\end{array}$ & $\begin{array}{c}\text { Percent of } \\
\text { diagnoses (\%) }\end{array}$ \\
\hline Autosomal dominant & 27 & 48.4 \\
De novo & 2 & \\
Inherited, parental mosaicism & 4 & \\
Inherited, no mosaicism & 11 & \\
Inheritance unknown & 37 & 40.7 \\
\hline Autosomal recessive & 26 & \\
\hline $\begin{array}{l}\text { Compound heterozygous } \\
\text { single-nucleotide variants }\end{array}$ & 11 & \\
\hline Homozygous & 1 \\
\hline Homozygous, uniparental disomy & 7 \\
\hline X-linked & 2 \\
\hline $\begin{array}{l}\text { De novo } \\
\text { Mitochondrial }\end{array}$ & 3 \\
\hline Dual diagnoses & 12 (6 cases) \\
\hline Autosomal dominant + autosomal dominant & 6 (3 cases) \\
\hline Autosomal recessive + autosomal recessive & 2 (1 case) \\
\hline Autosomal dominant + autosomal recessive & 4 (2 cases) \\
\hline
\end{tabular}

Uniparental disomy of chromosome 9 led to a homozygous SIGMAR1 mutation and juvenile amyotrophic lateral sclerosis in case 25. Parental consanguinity, reported in 4.5\% (22/486) of all adult cases, was overrepresented among those with diagnoses based on homozygous variants $(5 / 10,50 \%)$ and autosomal recessive diagnoses $(5 / 37,13.5 \%$, two-tailed $P=0.02$, Fisher exact test). Only recessive diagnoses were reported in cases with consanguineous parents. Three of seven X-linked diagnoses were in female patients; two of these were de novo diagnoses of Cornelia de Lange syndrome, caused by mutations in SMC1A or HDAC8 (cases 34 and 41).

\section{Phenotypes}

Each individual's phenotype as provided by the referring physician was completely represented by an average of $10 \mathrm{HPO}$ terms. HPO terms representing neurologic and developmental disorders were frequent: the terms "motor delay," "intellectual disability," "seizures," and "delayed speech and language development" each occurred in more than 100 of the 486 cases (Table 3). To evaluate the overall phenotypic composition of this patient population, cases were assigned to one or more of 22 HPO phenotype classes based on their HPO terms. Neoplasmrelated HPO terms were always assigned to the neoplasm class. Abnormalities of the nervous system, musculature, and skeletal system were the most frequent phenotype classes (Figure 2a).

To better understand intellectual disability (ID) in adults, we analyzed the subset of cases with the HPO term "neurodevelopmental abnormality" or one of several daughter terms. Of 53 such individuals with molecular diagnoses, 66.1\% (37/56) of their diagnoses were autosomal dominant (Supplementary Table S1 online), although in five cases the diagnosis explained
Table 3 Human Phenotype Ontology terms occurring in more than $10 \%$ of adult exome cases

\begin{tabular}{lcc} 
HPO term & $\begin{array}{c}\text { Number of } \\
\text { occurrences }\end{array}$ & $\begin{array}{c}\text { Percent } \\
\text { of cases (\%) }\end{array}$ \\
\hline Motor delay & 123 & 25.3 \\
\hline Intellectual disability & 121 & 24.9 \\
\hline Seizures & 113 & 23.3 \\
\hline Delayed speech and language development & 110 & 22.6 \\
\hline Abnormality of movement & 78 & 16.0 \\
\hline Spasticity & 78 & 16.0 \\
\hline Hypertonia & 78 & 16.0 \\
\hline Ataxia & 75 & 15.4 \\
\hline Scoliosis & 68 & 14.0 \\
\hline Muscular hypotonia & 64 & 13.2 \\
\hline Abnormality of brain morphology & 63 & 13.0 \\
\hline Abnormal face shape & 62 & 12.8 \\
\hline Joint hypermobility & 60 & 12.3 \\
\hline Short stature & 58 & 11.9 \\
\hline Abnormality of the eye & 58 & 11.9 \\
\hline Abnormality of the skin & 51 & 10.5 \\
\hline
\end{tabular}

components of the phenotype but not the ID. De novo mutations were reported in $75.0 \%$ (24/32) of autosomal dominant ID diagnoses. One of the remaining eight cases exhibited evidence of parental mosaicism; parental samples were unavailable in six cases.

Diagnostic rate was dependent on phenotype by a Monte Carlo analysis of 10,000 randomizations of diagnosis status $(P$ $=0.0053$ ). Diagnostic rates within each phenotype class varied, with abnormalities of the nervous system, skeletal system, musculature, head and neck, and growth trending toward higher diagnostic rates, suggesting the presence of these features can portend a favorable impact on establishing a diagnosis using WES (Figure 2b). Conversely, abnormalities of the abdomen, genitourinary, hematologic, immune, and respiratory systems, as well as oncologic findings, were associated with a reduced diagnostic rate. The highest diagnostic rate was seen in subjects with neurodevelopmental abnormalities (27.7\%, 53/191).

A family history overlapping with part or all of the proband's phenotype did not affect the molecular diagnosis rate, with a $15.5 \%(34 / 219)$ diagnostic rate among cases with a positive family history not significantly different from the $19.1 \%$ (51/267) diagnostic rate in cases with a negative family history (two-tailed $P=0.34$, Fisher exact test). In six cases reporting a positive family history, WES was also performed on an affected sibling (four cases) or parent (two cases), but no molecular diagnosis was reported.

\section{Secondary findings}

Seven medically actionable findings meeting ACMG criteria for secondary findings ${ }^{24}$ were reported in six adult probands $(1.2 \%, 6 / 482,95 \%$ CI: $0.25-2.23 \%)$, including one patient with 


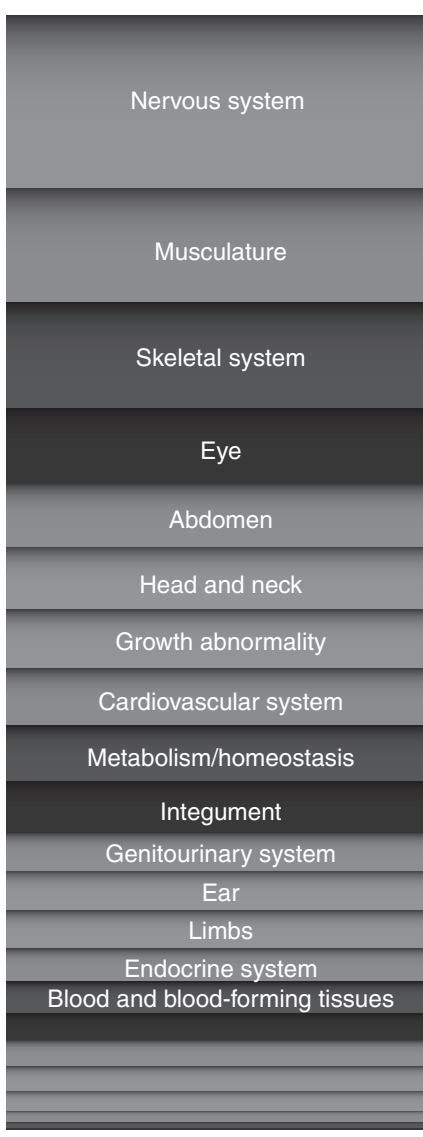

b



Immune system

Connective tissue

Respiratory system

Neoplasm

Prenatal development, birth

Voice

Breast

Figure 2 Phenotypic spectrum of individuals undergoing whole-exome sequencing. (a) Scaled representation of relative frequency of each phenotype class within this series. Individual cases may be counted in multiple classes. (b) Diagnostic rate for each phenotype class (gray bars, left y-axis) and percent of all cases for each phenotype class (blue line, right y-axis). ${ }^{*}$ Monte Carlo $P<0.05$ for the association between diagnostic rate and phenotype class.

both a pathogenic BRCA1 mutation, previously identified in her mother, and a deleterious DSC2 mutation associated with arrhythmogenic right ventricular cardiomyopathy. ${ }^{25}$ There was a significantly higher frequency of ACMG secondary findings in pediatric probands tested during the same interval (3.1\%, $114 / 3,648$, two-tailed $P=0.020$, Fisher exact test). The Whole Genome Laboratory reported a medically actionable finding outside the ACMG guidelines ${ }^{11}$ in six adult patients $(1.2 \%$, 6/481), including two mitochondrial gene mutations associated with increased risk of aminoglycoside-induced nonsyndromic hearing loss, two cardiomyopathy genes ( $A B C C 9, A K N R D 1$ ), one cancer susceptibility gene (RAD51D), and one novel lossof-function mutation in a cholesterol metabolism gene $(A P O B$, for which ACMG recommends reporting only known pathogenic mutations). Concurrently, $1.3 \%(49 / 3,648)$ of pediatric probands had medically actionable findings outside the current ACMG gene list (two-tailed $P=1$, Fisher exact test). Carrier status for autosomal recessive conditions was reported in 24 of 486 cases of disorders recommended for testing as part of reproductive planning by the $\mathrm{ACMG}^{26}$ : cystic fibrosis, TaySachs disease, Canavan disease, Gaucher disease, sickle cell anemia, and Niemann-Pick disease type A.

\section{DISCUSSION}

Clinical WES in adult patients had a diagnostic rate of $17.5 \%$ in our series, which is significantly lower than $25.2 \%$ previously reported in a primarily pediatric population (two-tailed $P=0.0003$, Fisher exact test). ${ }^{11}$ Within this cohort of adult patients referred for WES, a positive family history was not predictive of molecular diagnosis, and molecular diagnoses often resulted from de novo events, informing the structure of Mendelian diseases in adults. The association of family history with diagnosis rate may be impacted by the underlying clinical phenotypes in these adult patients referred for WES. For example, cancer diagnoses were a rare indication for WES in this case population. Inheritance patterns of the diagnoses were similar to the primarily pediatric population. ${ }^{11}$ We observed an unexpectedly high prevalence of de novo mutations, which may be driven by the high frequency of neurodevelopmental abnormalities in this series. Like that observed in a pediatric population, three of seven X-linked diagnoses were X-linked dominant traits in females. ${ }^{11}$ The small number but higher rate of mitochondrial diagnoses (3/86) compared to a contemporary primarily pediatric population ${ }^{11}(1 / 504)$ may represent lack of available mitochondrial analyses when these adults 
were initially evaluated as children. Small insertion or deletion variants, which are less robustly detected by WES analysis algorithms, ${ }^{27}$ comprise nearly $25 \%$ of disease-causing variants in both pediatric and adult series, ${ }^{11}$ underscoring the need for improved detection and genotyping of these variants. We anticipate that implementation of the most updated ACMG guidelines ${ }^{28}$ for variant interpretation may allow improved reporting of likely pathogenic variants and evidence-based assessment of loss-of-function variants in WES reporting. The clinical utility of molecular diagnosis through WES in this adult population is demonstrated through medical management recommendations, anticipatory guidance, and provision of recurrence risk for patients and families, as recently described by the ACMG. ${ }^{10}$

The diagnostic indications in this adult case series were predominantly neurologic, for example, $39.3 \%$ with neurodevelopmental delay, perhaps reflecting the specialty of ordering physicians. However, this phenotypic spectrum differed markedly from that within adult genetics clinics. ${ }^{11,14,15,29}$ For example, the $9.7 \%$ of adult WES cases with oncologic phenotypes is less than the $35 \%$ in adult genetics clinic, ${ }^{29}$ likely reflecting both the well-defined nature of many familial cancer syndromes and the availability of comprehensive next-generation hereditary cancer gene panels. Neurologic (14\%) and cardiovascular (13\%) phenotypes represent the next most common indications in adult genetics clinic, whereas 81 and $27 \%$ of adult WES cases, respectively, have such phenotypes. These differences illustrate the selective use of WES in the adult genetics clinic, in addition to its increasing use in adult neurology and neurogenetics centers. As the number of disease-gene and disease-variant associations continues to grow, combined with reporting of clinical sequencing studies from different patient populations (see for example https://cser-consortium.org), we will gain improved knowledge regarding which adult patients are most appropriate for WES as a clinical test across the medical spectrum.

Diagnoses were more common among individuals with specific phenotypes, including abnormalities of the head and neck, skeletal system, musculature, nervous system, or growth. Exploration of the relationship of pairwise combinations of phenotypes to the likelihood of molecular diagnosis did not find significant differences for specific pairs of anomalies in this cohort, although larger studies may be needed to investigate this issue with sufficient power.

The "clan genomics" hypothesis for human disease traits posits that variants arising in the proband (i.e., de novo mutations), or a recent antecedent in the family or clan, are important to disease trait manifestation. ${ }^{30}$ Such de novo or recent events can cause dominant and X-linked diagnoses or autosomal recessive diagnoses in the setting of consanguinity, which allows for rapid attainment of homozygosity for newly occurring variants within the family or clan. Although no de novo recessive variants were present in our cohort, homozygous variants accounted for $29.7 \%$ of autosomal recessive diagnoses, and 7/11 homozygous variants were novel. Parental consanguinity was reported in $50 \%$ of homozygous mutations not explained by uniparental disomy. The high proportion of homozygous novel variants in autosomal recessive diagnoses along with parental consanguinity support the potential role of recently emerging variants in recessive disease.

We further observed pathogenic de novo events in $28.6 \%$ (2/7) of X-linked and $61.4 \%$ (27/44) of autosomal dominant diagnoses, all reported in adults younger than 30 years, including two de novo diagnoses (PRICKLE2, CREBBP) in one individual with ID and seizures (Supplementary Table S1 online). Importantly, when parental samples were available, de novo events were detected in $81.8 \%$ (27/33) of dominant diagnoses, which is similar to the $86.7 \%(208 / 240)$ reported in a primarily pediatric population. ${ }^{11}$ The diagnostic rate for autosomal dominant disease is impacted by parental sample availability, particularly for missense mutations not previously documented to be disease-associated. We report 24 missense variants among 44 total autosomal dominant diagnoses (55\%), 13 of which were novel. Determination of pathogenicity for these novel missense variants required parental samples, because $85 \%$ (11/13) were de novo, one was inherited from an affected mother, and one was from an unaffected mosaic father. These findings suggest that the lower diagnostic rate in individuals older than 30 years of age may be explained, at least in part, by limited parental sample availability. Overall, these data demonstrate that interpretation of novel missense variants as pathogenic frequently relies on de novo status in autosomal dominant disorders and support a role for trio-WES for detection of de novo variants. That de novo mutations underlie a high proportion of genetic disease in adults is unexpected and illustrates that physicians who rely on a positive family history to refer for genetic testing may miss such diagnoses.

The majority of individuals with de novo diagnoses (26/29) had disorders that included neurodevelopmental delay with onset in childhood, representing 13.6\% (26/191) of all cases involving neurodevelopmental delay and providing a molecular explanation for the developmental phenotype in 24 of these. These findings are similar to prior reports that between $16 \%^{31}$ and $55 \%{ }^{32}$ of pediatric ID cases, and $16 \%$ of a variety of pediatric developmental disorders, ${ }^{33}$ have de novo autosomal dominant molecular diagnoses by trio-WES. This suggests greater phenotypic overlap with pediatric genetics cases among these individuals with de novo mutations than is present in the adult WES cases as a whole. Additionally, more than half of these diagnoses were made in genes with disease associations discovered in the past decade. Therefore, the ascertainment of frequent de novo mutations in our adult cohort is potentially attributable to both limited genetic diagnostic testing capabilities and limited disease gene knowledge when these individuals initially presented as children.

Although phenotypic similarity between young adults presenting for WES and pediatric cases potentially contributes to increased diagnoses in younger adults (Figure 1), other factors contribute to a lower overall diagnostic rate in adults. Interestingly, recurrent molecular diagnoses (diagnostic variants found in the same gene in multiple subjects) were observed in only $10.6 \%(9 / 85)$ of molecularly diagnosed adult cases 
compared to $56.0 \%$ of cases in a primarily pediatric population. ${ }^{11}$ These findings may reflect the smaller number of adult cases, but may also indicate a greater diversity of underlying genetic disorders in adults.

Diagnosis of adults with genetic disease provides a unique challenge. Effects of environmental exposures or signs of more common/complex non-Mendelian medical disease may obscure adult phenotypes. Lack of parental samples may limit recognition of de novo variants, and adult phenotypes may be milder, more heterogeneous, and with variable onset, possibly due to rare hypomorphic alleles. Other limitations of WES, such as difficulties in detecting copy-number variant and repeat expansion, are magnified in adult patients for whom chromosomal microarray or repeat expansion analysis may not have been performed prior to WES. The greater clinical availability of molecular diagnostic tools such as WES has allowed diagnoses that were not possible 10 or even 5 years ago, but this same success underscores the need for a more complete interrogation of individual genomic variation, particularly rare variant alleles and copy-number variation, as these both contribute to adult disease. $^{34}$

Despite the present limitations and clear areas for further discovery (gene function identification), WES is a clinically useful diagnostic tool with the ability to deconvolute complicated adult phenotypic presentations and diagnose exceedingly rare conditions. This advantage is evident in several of the cases described in Supplementary Table S1 online, such as the rare diagnosis of glutamate formiminotransferase deficiency (OMIM 229100), Sotos syndrome in an individual with an atypical phenotype not ascertained in childhood (OMIM 117550), blended phenotypes due to coexistence of multiple genetic conditions, ${ }^{11,12}$ and molecular diagnoses such as myotonia congenita (OMIM 255700) and Kufor-Rakeb syndrome (OMIM 606693) that inform clinical management of symptoms (Supplementary Table S1 online). ${ }^{35,36}$

Identification of secondary, potentially actionable mutations provides opportunities to inform medical surveillance and disease prevention. ${ }^{37}$ The ACMG recommends actively searching for such actionable findings in all cases undergoing clinical WES, ${ }^{24}$ and this practice will probably increase quality of life and be cost-effective. ${ }^{38}$ We identified findings in $1.2 \%$ of adults, similar to that previously reported in adult controls $(92 / 6,503,1.4 \%, P=1.00)^{39}$ but significantly lower than the $3.1 \%$ frequency in pediatric cases in our clinical laboratory $(P=0.020) .{ }^{11}$ Differences may reflect the elimination of secondary findings in adults who have already manifested features (or had a positive family history) related to these later-onset conditions than children. ${ }^{40}$

We report experiences with WES as a diagnostic tool in an adult population referred to an academic diagnostic laboratory, a group for which phenotype heterogeneity, aging, and environmental exposures increase the diagnostic challenges. The adult patients referred for WES were phenotypically similar to pediatric cohorts, with a predominance of developmental or neurologic disorders. ${ }^{11}$ Molecular diagnoses were more common in young adults. The observation of two molecular genetic diagnoses in approximately $7 \%$ of those with diagnoses is similar to findings from our previous studies of $6.5 \%{ }^{12}$ and $4.6 \% .{ }^{11}$ Molecular diagnoses and medically actionable secondary findings provided anticipatory information, management and surveillance guidance, and, in some cases, disease-specific treatment options that may result in a significant improvement in quality of life. The presence of a positive family history did not predict molecular diagnosis, and the high contribution of de novo events potentially informs the Mendelian basis of genetic disease in adults. Moreover, the paucity of recurrent molecular diagnoses suggests that much remains to be learned about the underlying genes and genetic architecture of adult genetic disease.

\section{SUPPLEMENTARY MATERIAL}

Supplementary material is linked to the online version of the paper at http://www.nature.com/gim

\section{ACKNOWLEDGMENTS}

J.E.P. was supported by the Medical Genetics Research Fellowship Program NIH/NIGMS NIH T32 GM07526. W.W. was supported by the Career Development Award K23NS078056 from the National Institute of Neurological Disorders and Stroke (NINDS). This work was funded in part by grants U01 HG006485 (S.E.P.) from the National Human Genome Research Institute (NHGRI) and National Cancer Institute (NCI), U54 HG006542 (J.R.L.) from the NHGRI and National Heart, Lung, and Blood Institute (NHLBI), U54-HG003273 (R.A.G.) from the NHGRI, and R01 NS058529 (J.R.L.) from the NINDS. $\mathrm{NHGRI}, \mathrm{NCl}$, and NINDS had no role in the design and conduct of the study; the collection, management, analysis, and interpretation of the data; the preparation, review, or approval of the manuscript; or the decision to submit the manuscript for publication.

\section{DISCLOSURE}

Baylor College of Medicine (BCM) and Miraca Holdings Inc. have formed a joint venture with shared ownership and governance of the Baylor Miraca Genetics Laboratories (BMGL), which performs clinical exome sequencing. J.A.R., Z.N., F.X., R.E.P., M.W., A.L.B., C.M.E., Y.Y., R.A.G., J.R.L., and S.E.P. are employees of BCM and derive support through a professional services agreement with the BMGL. S.E.P. and J.R.L. serve on the Scientific Advisory Board of the BMGL. R.A.G. serves as interim Chief Scientific Officer of the BMGL. A.L.B. serves as Chief Medical Officer of the BMGL. M.B. is the founder of Codified Genomics Inc., and derives personal fees from Illumina Inc. S.D. is the CEO and cofounder of PanGenomics Clinical Genetics Center in India. J.A.R. reports personal fees from Signature Genomic Laboratories, PerkinElmer, Inc., in the past 36 months. R.A.G. reports consulting fees from GE-Clarient. J.R.L. has stock ownership in 23andMe, is a paid consultant for Regeneron Pharmaceuticals, has stock options in Lasergen, Inc., and is a coinventor of US and European patents related to molecular diagnostics for inherited neuropathies, eye diseases, and bacterial genomic fingerprinting. The other authors declare no conflict of interest. 


\section{REFERENCES}

1. Wheeler DA, Srinivasan M, Egholm M, et al. The complete genome of an individual by massively parallel DNA sequencing. Nature 2008;452:872-876.

2. Levy S, Sutton G, Ng PC, et al. The diploid genome sequence of an individual human. PLoS Biol 2007; 5:e254.

3. Albert TJ, Molla MN, Muzny DM, et al. Direct selection of human genomic loci by microarray hybridization. Nat Methods 2007;4:903-905.

4. Bainbridge $M N$, Wang $M$, Burgess $D L$, et al. Whole exome capture in solution with $3 \mathrm{Gbp}$ of data. Genome Bio/ 2010;11:R62.

5. Lupski JR, Reid JG, Gonzaga-Jauregui C, et al. Whole-genome sequencing in a patient with Charcot-Marie-Tooth neuropathy. N Engl J Med 2010;362: 1181-1191.

6. Boyden LM, Choi M, Choate KA, et al. Mutations in kelch-like 3 and cullin 3 cause hypertension and electrolyte abnormalities. Nature 2012;482:98-102.

7. Worthey EA, Mayer AN, Syverson GD, et al. Making a definitive diagnosis: successful clinical application of whole exome sequencing in a child with intractable inflammatory bowel disease. Genet Med 2011;13:255-262.

8. Iglesias A, Anyane-Yeboa K, Wynn J, et al. The usefulness of whole-exome sequencing in routine clinical practice. Genet Med 2014;16:922-931.

9. Wangler MF, Gonzaga-Jauregui C, Gambin T, et al.; Baylor-Hopkins Center for Mendelian Genomics. Heterozygous de novo and inherited mutations in the smooth muscle actin (ACTG2) gene underlie megacystis-microcolon-intestinal hypoperistalsis syndrome. PLoS Genet 2014;10:e1004258.

10. ACMG Board of Directors. Clinical utility of genetic and genomic services: a position statement of the American College of Medical Genetics and Genomics. Genet Med 2015;17:505-507.

11. Yang Y, Muzny DM, Xia F, et al. Molecular findings among patients referred for clinical whole-exome sequencing. JAMA 2014;312:1870-1879.

12. Yang Y, Muzny DM, Reid JG, et al. Clinical whole-exome sequencing for the diagnosis of mendelian disorders. N Engl J Med 2013;369:1502-1511.

13. Bainbridge MN, Hu H, Muzny DM, et al. De novo truncating mutations in ASXL3 are associated with a novel clinical phenotype with similarities to Bohring-Opitz syndrome. Genome Med 2013;5:11.

14. Farwell KD, Shahmirzadi L, El-Khechen D, et al. Enhanced utility of familycentered diagnostic exome sequencing with inheritance model-based analysis: results from 500 unselected families with undiagnosed genetic conditions. Genet Med 2015;17:578-586.

15. Lee $\mathrm{H}$, Deignan JL, Dorrani $\mathrm{N}$, et al. Clinical exome sequencing for genetic identification of rare Mendelian disorders. JAMA 2014;312:1880-1887.

16. Li C, Szybowska M. A novel mutation C.4003 G>C in the CREBBP gene in an adult female with Rubinstein-Taybi syndrome presenting with subtle dysmorphic features. Am J Med Genet A 2010;152A:2939-2941.

17. Prada CE, Gonzaga-Jauregui C, Tannenbaum R, et al. Clinical utility of wholeexome sequencing in rare diseases: Galactosialidosis. Eur J Med Genet 2014; $57: 339-344$.

18. Collison FT, Xie YA, Gambin T, et al. Whole exome sequencing identifies an adult-onset case of methylmalonic aciduria and homocystinuria type $C$ (cblC) with non-syndromic bull's eye maculopathy. Ophthalmic Genet 2015;36: 270-275

19. Gonzalez-Garay ML, McGuire AL, Pereira S, Caskey CT. Personalized genomic disease risk of volunteers. Proc Natl Acad Sci USA 2013;110:16957-16962.

20. Caskey CT, Gonzalez-Garay ML, Pereira S, McGuire AL. Adult genetic risk screening. Annu Rev Med 2014;65:1-17.

21. Bainbridge MN, Wang M, Wu Y, et al. Targeted enrichment beyond the consensus coding DNA sequence exome reveals exons with higher variant densities. Genome Biol 2011:12:R68.

22. Richards CS, Bale S, Bellissimo DB, et al.; Molecular Subcommittee of the ACMG Laboratory Quality Assurance Committee. ACMG recommendations for standards for interpretation and reporting of sequence variations: Revisions 2007. Genet Med 2008;10:294-300.

23. Köhler S, Doelken SC, Mungall CJ, et al. The Human Phenotype Ontology project: linking molecular biology and disease through phenotype data. Nucleic Acids Res 2014;42(Database issue):D966-D974.

24. Green RC, Berg JS, Grody WW, et al.; American College of Medical Genetics and Genomics. ACMG recommendations for reporting of incidental findings in clinical exome and genome sequencing. Genet Med 2013;15:565-574.

25. Beffagna $G$, De Bortoli M, Nava A, et al. Missense mutations in desmocollin-2 $\mathrm{N}$-terminus, associated with arrhythmogenic right ventricular cardiomyopathy, affect intracellular localization of desmocollin-2 in vitro. BMC Med Genet 2007;8:65.

26. Gross SJ, Pletcher BA, Monaghan KG; Professional Practice and Guidelines Committee. Carrier screening in individuals of Ashkenazi Jewish descent. Genet Med 2008;10:54-56.

27. DePristo MA, Banks E, Poplin R, et al. A framework for variation discovery and genotyping using next-generation DNA sequencing data. Nat Genet 2011:43:491-498.

28. Richards S, Aziz N, Bale S, et al. Standards and guidelines for the interpretation of sequence variants: a joint consensus recommendation of the American College of Medical Genetics and Genomics and the Association for Molecular Pathology. Genet Med 2015;17:405-424.

29. Eble TN, Nagamani SC, Franco LM, Plon SE, Blazo M, Dhar SU. The practice of adult genetics: a 7-year experience from a single center. Am J Med Genet A 2013;161A:89-93.

30. Lupski JR, Belmont JW, Boerwinkle E, Gibbs RA. Clan genomics and the complex architecture of human disease. Cell 2011;147:32-43.

31. de Ligt J, Willemsen $\mathrm{MH}$, van Bon BW, et al. Diagnostic exome sequencing in persons with severe intellectual disability. N Engl J Med 2012;367: 1921-1929.

32. Rauch A, Wieczorek D, Graf E, et al. Range of genetic mutations associated with severe non-syndromic sporadic intellectual disability: an exome sequencing study. Lancet 2012;380:1674-1682.

33. Wright CF, Fitzgerald TW, Jones WD, et al.; DDD study. Genetic diagnosis of developmental disorders in the DDD study: a scalable analysis of genome-wide research data. Lancet 2015;385:1305-1314.

34. DiVincenzo C, Elzinga CD, Medeiros AC, et al. The allelic spectrum of CharcotMarie-Tooth disease in over 17,000 individuals with neuropathy. Mol Genet Genomic Med 2014;2:522-529.

35. Statland JM, Bundy BN, Wang Y, et al.; Consortium for Clinical Investigation of Neurologic Channelopathies. Mexiletine for symptoms and signs of myotonia in nondystrophic myotonia: a randomized controlled trial. JAMA 2012;308: 1357-1365.

36. Williams DR, Hadeed A, al-Din AS, Wreikat AL, Lees AJ. Kufor Rakeb disease: autosomal recessive, levodopa-responsive parkinsonism with pyramidal degeneration, supranuclear gaze palsy, and dementia. Mov Disord 2005;20:1264-1271.

37. Green RC, Lupski JR, Biesecker LG. Reporting genomic sequencing results to ordering clinicians: incidental, but not exceptional. JAMA 2013;310:365-366.

38. Bennette CS, Gallego CJ, Burke W, Jarvik GP, Veenstra DL. The cost-effectiveness of returning incidental findings from next-generation genomic sequencing. Genet Med 2015;17:587-595.

39. Amendola LM, Dorschner MO, Robertson PD, et al. Actionable exomic incidental findings in 6503 participants: challenges of variant classification. Genome Res 2015:25:305-315.

40. Jurgens J, Ling $\mathrm{H}$, Hetrick K, et al. Assessment of incidental findings in 232 whole-exome sequences from the Baylor-Hopkins Center for Mendelian Genomics. Genet Med 2015;17:782-788. 